\title{
A SUM CONNECTED WITH THE PARTITION FUNCTION
}

\section{ALBERT LEON WHITEMAN}

1. Introduction. In the formula for the number $p(n)$ of unrestricted partitions of an integer $n$ there appears the sum [1] ${ }^{1}$

$$
A_{k}(n)=\sum_{h \bmod k}^{\prime} \omega_{h, k} \exp (-2 \pi i h n / k)
$$

where the dash ' beside the summation symbol indicates here and in the sequel that the letter of summation runs only through a reduced residue system with respect to the modulus. The symbol $\omega_{h, k}$ denotes certain $24 k$ th roots of unity given by

$$
\omega_{h, k}=\exp (\pi i s(h, k)) \text {, }
$$

where $s(h, k)$ is a Dedekind sum [2] defined by

$$
s(h, k)=\sum_{\mu=1}^{k}\left(\left(\frac{\mu}{k}\right)\right)\left(\left(\frac{\mu h}{k}\right)\right) .
$$

The symbol $((x))$, in turn, is defined as follows:

$$
((x))=\left\{\begin{array}{lr}
x-[x]-1 / 2 & \text { for } x \text { not an integer, } \\
0 & \text { for } x \text { an integer, }
\end{array}\right.
$$

where $[x]$ denotes, as usual, the greatest integer not exceeding $x$. D. H. Lehmer [3] has investigated these sums on the basis of a different expression for the roots of unity involved. In the first place he factored the $A_{k}(n)$ according to the prime powers contained in $k$. Secondly, by reducing them to sums studied by $\mathrm{H}$. D. Kloosterman [4] and H. Salié [5], he evaluated the $A_{k}(n)$ explicitly in the case in which $k$ is a prime or a power of a prime. Both results together provide a method for calculating the $A_{k}(n)$. Alternate proofs of Lehmer's factorization theorems have been given in [2]. In the present note a new approach to the second of Lehmer's results is presented. The method given here is simpler than Lehmer's method, especially in the treatment of the case $k=2^{\lambda}$.

2. Some lemmas. The proofs are based in part on three lemmas, which occur as Theorems 17, 18, 19 in [2].

LEMMA 1. Let $\theta=\theta(k)$ denote 1 for $3 \nmid k$ and 3 for $3 \mid k$ so that $\theta k$ is

Presented to the Society, September 5, 1941; received by the editors June 20, 1946.

1 Numbers in brackets refer to the bibliography at the end of the paper. 
either prime to 3 or divisible by $3^{2}$. For $(h, k)=1$ we have

$$
12 h k s(h, k) \equiv h^{2}+1(\bmod \theta k) \text {. }
$$

Moreover

$$
12 k s(h, k)=0(\bmod 3)
$$

if and only if $3 \nmid k$.

Lemma 2. For odd $k$ we have $12 k s(h, k) \equiv k+1-2(h \mid k)(\bmod 8)$, where $(h \mid k)$ denotes the Legendre-Jacobi symbol.

LEMMA 3. If $k$ is equal to $2^{\lambda} j, \lambda \geqq 0$, and $j$ and $h$ are odd integers, then $12 h k s(h, k) \equiv h^{2}+k^{2}+3 k+1+2 k(k \mid h)\left(\bmod 2^{\lambda+3}\right)$.

The following lemmas concerning generalized Kloosterman sums are also needed.

LEMMa 4. Let $k=p^{\alpha}$, where $p$ is an odd prime. Put

$$
S_{k}(n)=\sum_{h \bmod k}^{\prime}(h \mid k) \exp (2 \pi i(n h+\bar{h}) / k),
$$

where $\bar{h}$ is defined as any solution of the congruence $h \bar{h} \equiv 1(\bmod k)$. Then $S_{k}(n)= \begin{cases}0 & \text { if } n \text { is a non-residue of } k \text { prime to } k, \\ 2 i^{((k-1) / 2)^{2}} k^{1 / 2} \cos 4 \pi m / k & \text { if } m^{2} \equiv n(\bmod k), n \text { prime to } k, \\ 0 & \text { if } n \text { is divisible by } p \text { and } \alpha>1, \\ i^{((k-1) / 2)^{2} k^{1 / 2}} & \text { if } n \text { is divisible by } p \text { and } \alpha=1 .\end{cases}$

LEMMA 5. Let $k=p^{\beta}, \beta>1$, and $n \equiv 1(\bmod p)$, where $p$ denotes an odd prime congruent to $3(\bmod 4)$. Then

$$
\begin{aligned}
\sum_{h \bmod k}^{\prime}\left(h \mid p^{\beta-1}\right) \exp & (2 \pi i(n h+\bar{h}) / k) \\
= & 2 i^{((k-1) / 2)^{2}+1}(m \mid p) k^{1 / 2} \sin (4 \pi m / k),
\end{aligned}
$$

where $m$ is an integer such that $m^{2} \equiv n(\bmod k)$.

Lemmas 4 and 5 may be proved by employing a method due to the author [6]. The method carries over step by step with only slight modifications and it does not seem necessary to present it again here.

LEMma 6. If $k$ is equal to $2^{\lambda}, \lambda \geqq 9$, and $n$ is an odd integer, then

$$
\begin{aligned}
\sum_{h \bmod k}^{\prime}(2 k \mid h) \exp (2 \pi i n & (h+\bar{h}) / k) \\
& =2(k \mid n)(2 k)^{1 / 2} \cos \left(\pi / 4+(-1)^{(n-1) / 2} 4 \pi n / k\right) .
\end{aligned}
$$


Lemma 6 may be established easily if use is made of Salie's [5] discussion ( $\$ 3$ ) of the corresponding sum without a quadratic character. In fact, the introduction of the character has no influence on the argument. It is therefore not necessary to reproduce the proof here.

3. Proof of Lehmer's theorems. In order to apply the lemmas of the preceding section to the evaluation of $A_{k}(n)$ for $k$ a power of an odd prime $p$, we divide the discussion into two cases according as $p>3$ or $p=3$. For $p>3$ we employ the congruence

$$
\begin{aligned}
6 k\left[-((k-1) / 2)^{2}-1+(2 h \mid k)\right] & +24^{2} a h+24 \cdot \overline{24} \bar{h} \\
& \equiv 12 k s(h, k)-24 h n(\bmod 24 k),
\end{aligned}
$$

where $a$ and $\bar{h}$ are defined by means of the congruences

$$
24^{2} a \equiv 1-24 n(\bmod k), \quad h \bar{h} \equiv 1(\bmod k) .
$$

Divisibility with respect to $k$ and with respect to 3 in congruence (2) follows at once from Lemma 1. Divisibility with respect to 8 follows from Lemma 2 and the well known relation $(2 \mid k)=(-1)^{\left(k^{2}-1\right) / 8}$. Hence, from (1) and (2), we get

$$
\begin{aligned}
A_{k}(n) & =\sum_{h \bmod k}^{\prime} \exp [2 \pi i(k s(h, k) / 2-h n) / k] \\
& =\sum_{h \bmod k}^{\prime} \exp \left\{2 \pi i \left[k\left(-((k-1) / 2)^{2}-1+(2 h \mid k)\right) / 4\right.\right. \\
& =(-i)^{((k-1) / 2)^{2}}(2 \mid k) \sum_{h \bmod k}^{\prime}(h \mid k) \exp [2 \pi i(24 a h+\overline{24} \bar{h}) / k] \\
& =(-i)^{((k-1) / 2)^{2}}(3 \mid k) \sum_{h \bmod }^{\prime}(h \mid k) \exp [2 \pi i(a h+\bar{h}) / k] .
\end{aligned}
$$

Applying Lemma 4, and using (3), we obtain the following theorem.

THEOREM 1. If $k=p^{\alpha}, \alpha \geqq 1$, and $v=1-24 n$, where $p$ is a prime greater than 3 , then

$$
A_{k}(n)=\left\{\begin{array}{lr}
0 & \text { if } v \text { is a non-residue of } k, \text { prime to } k, \\
2(3 \mid k) k^{1 / 2} \cos (4 \pi m / k) & \text { if } v \equiv(24 m)^{2}(\bmod k), \text { prime to } k, \\
0 & \text { if } v \equiv 0(\bmod p) \text { and } \alpha>1, \\
(3 \mid k) k^{1 / 2} & \text { if } v \equiv 0(\bmod p) \text { and } \alpha=1 .
\end{array}\right.
$$

We turn next to the case which arises when $k=p^{\alpha}$ and $p=3$. This

2 This condition should not be confused with $(v \mid k)=-1$. We mean that no solution exists of the congruence $x^{a} \equiv v(\bmod k)$. 
time we use the congruence

$$
\begin{array}{r}
6 k\left[-((k-1) / 2)^{2}-1+(2 h \mid k)\right]+8^{2} a h+8 \cdot \overline{8} \bar{h} \\
\equiv 12 k s(h, k)-24 h n(\bmod 24 k),
\end{array}
$$

where $a$ and $\hbar$ are defined by the congruences

$$
8^{2} a \equiv 1-24 n(\bmod 3 k), \quad h \bar{h} \equiv 1(\bmod 3 k) .
$$

Divisibility with respect to $3 k$ in congruence (4) follows immediately from Lemma 1. Divisibility with respect to 8 follows from Lemma 2. Hence, by (1) and (4), we have

$$
\begin{aligned}
& A_{k}(n)=\sum_{h \bmod k}^{\prime} \exp [2 \pi i(3 k s(h, k) / 2-3 h n) / 3 k] \\
& =\sum_{h \bmod k}^{\prime} \exp \left\{2 \pi i \left[3 k\left(-((k-1) / 2)^{2}-1+(2 h \mid k)\right) / 4\right.\right. \\
& +8 a h+\overline{8} \bar{h}] / 3 k\}
\end{aligned}
$$

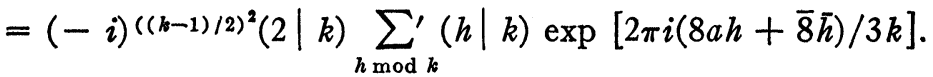

As $h$ runs through a reduced residue system $\bmod k$, so does $h+k$. Replace $\bar{h}$ by $\bar{h}-k \bar{h}^{2}$, and observe that (5) implies that

$$
8 a(h+k)+\overline{8}\left(\bar{h}-k \bar{h}^{2}\right) \equiv 8 a h+\overline{8} \bar{h} \quad(\bmod 3 k) .
$$

If $h$ now runs over a reduced residue system $\bmod 3 k$, instead of $\bmod k$, we obtain $3 A_{k}(n)$ instead of $A_{k}(n)$. Therefore,

$$
\begin{aligned}
A_{k}(n) & =\frac{1}{3}(-i)^{((k-1) / 2)^{2}}(2 \mid k) \sum_{h \bmod 3 k}^{\prime}(h \mid k) \exp [2 \pi i(8 a h+\overline{8} \bar{h}) / 3 k] \\
& =\frac{1}{3}(-i)^{((k-1) / 2)^{2}} \sum_{h \bmod 3 k}^{\prime}(h \mid k) \exp [2 \pi i(a h+\bar{h}) / 3 k] .
\end{aligned}
$$

Since $a \equiv 1(\bmod 3)$, we may apply Lemma 5 with $\beta$ replaced by $\alpha+1$. Thus we get the following theorem.

THEOREM 2. If $k=3^{\beta}$, then

$$
A_{k}(n)=2(-1)^{\beta+1}(m \mid 3)(k / 3)^{1 / 2} \sin (4 \pi m / 3 k),
$$

where $m$ is an integer such that $(8 m)^{2} \equiv 1-24 n(\bmod 3 k)$.

Finally, we consider the case $k=2^{\lambda}$. For this purpose we use the congruence

(6) $3 \bar{h} k-6 \bar{h} k(k \mid h)+3^{2} a h+3 \cdot 3 \bar{h} \equiv 12 k s(h, k)-24 h n(\bmod 24 k)$, 
where $a$ and $\bar{h}$ are defined by the congruences

$$
3^{2} a \equiv 1-24 n(\bmod 8 k), \quad h \bar{h} \equiv 1(\bmod 8 k),
$$

and where we have assumed that $\lambda \geqq 3$.

Divisibility with respect to 3 in congruence (6) follows from the second part of Lemma 1 . Divisibility with respect to $8 k$ follows from Lemma 3. From (1) and (6) we now obtain

$$
\begin{aligned}
A_{k}(n) & =\sum_{h \bmod k}^{\prime} \exp [2 \pi i(4 k s(h, k)-8 h n) / 8 k] \\
& =\sum_{h \bmod k}^{\prime} \exp [2 \pi i(\bar{h} k-2 \bar{h} k(k \mid h)+3 a h+3 \bar{h}) / 8 k] \\
& =\frac{1}{8} \sum_{h \bmod 8 k}^{\prime} \exp [2 \pi i(h-2 h(k \mid h)) / 8] \exp [2 \pi i(3 a h+3 \bar{h}) / 8 k] .
\end{aligned}
$$

It is easy to verify that

$$
\exp [2 \pi i(h-2 h(k \mid h)) / 8]=(-1)^{\lambda+1}(k \mid 3 h) \exp [2 \pi i(3 h) / 8] .
$$

Therefore,

(8) $A_{k}(n)=\frac{1}{8}(-1)^{\lambda+1} \sum_{h \bmod 8 k}^{\prime}(k \mid 3 h) \exp [2 \pi i(3 a h+3 h(1+k)) / 8 k]$.

Now, for $\lambda \geqq 5$, we have $(1+k / 2)^{2} \equiv 1+k(\bmod 8 k)$. Furthermore, it follows from (7) that $a$ is a quadratic residue of $2^{\lambda+3}$ since $3^{2} a \equiv 1$ $(\bmod 8)$. Let $m^{2} \equiv a(\bmod 8 k)$. Replacing $h$ by $\overline{3} \bar{m}(1+k / 2) h$, and applying Lemma 6 , we obtain for $\lambda \geqq 6$,

$$
\begin{aligned}
A_{k}(n) & =\frac{1}{8}(-1)^{\lambda+1}(k \mid m) \sum_{h \bmod 8 k}^{\prime}(k \mid h) \exp [2 \pi i m(1+k / 2)(h+\bar{h}) / 8 k] \\
& =(-1)^{\lambda+1}(2 \mid m) k^{1 / 2} \cos \left\{\pi / 4+(-1)^{(m-1) / 2} 4 \pi[m(1+k / 2)] / 8 k\right) .
\end{aligned}
$$

The last equation may be simplified by employing the relation $(2 \mid m)=(-1)^{\left(m^{2}-1\right) / 8}$. We obtain thus the following theorem.

THEOREM 3. If $k=2^{\lambda}, \lambda \geqq 0$, then

$$
A_{k}(n)=(-1)^{\lambda}(-1 \mid m) k^{1 / 2} \sin (4 \pi m / 8 k),
$$

where $m$ is an integer such that $(3 m)^{2} \equiv 1-24 n(\bmod 8 k)$.

Actually we have established Theorem 3 only for $\lambda \geqq 6$. The verification of this theorem for $\lambda<6$ is left as an exercise for the reader. For $0 \leqq \lambda \leqq 3$, use the definition of $A_{k}(n)$ given by (1). For $\lambda=4,5$ use the formula for $A_{k}(n)$ given by (8). 


\section{BIBLIOGRAPHY}

1. H. Rademacher, On the partition function $p(n)$, Proc. London Math. Soc. (2) vol. 43 (1937) pp. 241-254.

2. H. Rademacher and A. L. Whiteman, Theorems on Dedekind sums, Amer. J. Math. vol. 63 (1941) pp. 377-407.

3. D. H. Lehmer, On the series for the partition function, Trans. Amer. Math. Soc. vol. 43 (1938) pp. 271-295.

4. H. D. Kloosterman, On the representation of numbers in the form $a x^{2}+b y^{2}+c z^{2}$ $+d t^{2}$, Acta Math. vol. 49 (1926) pp. 407-464.

5. H. Salié, Über die Kloostermanschen Summen $S(u, v ; q)$, Math. Zeit. vol. 34 (1932) pp. 91-109.

6. A. L. Whiteman, A note on Kloosterman sums, Bull. Amer. Math. Soc. vol. 51 (1945) pp. 373-377.

7. D. H. Lehmer, On a conjecture of Ramanujan, J. London Math. Soc. vol. 11 (1936) pp. 114-118.

Purdue University 